\title{
Trombolisi accelerata microsonica: trattamento innovativo della trombosi di una fistola artero-
} venosa nativa

\author{
Laura Buzzi ${ }^{1}$ Elena Alberghini ${ }^{1}$, Francesca Ferrario ${ }^{1}$, Ivano Baragetti ${ }^{1}$, Gaia Santagostino ${ }^{1}$, \\ Silvia Furiani ${ }^{1}$, Enzo Corghi ${ }^{1}$, Cristina Sarcina ${ }^{1}$, Veronica Terraneo ${ }^{1}$, Francesco Rastelli ${ }^{1}$, \\ Maria Cristina Gambirasio ${ }^{1}$, Giuseppe Bacchini², Claudio Pozzi ${ }^{1}$
}

\author{
${ }^{1}$ Nefrologia e Dialisi, Ospedale E. Bassini, Cinisello Balsamo (MI)
}

${ }^{2}$ Nefrologia e Dialisi, Ospedale A. Manzoni, Lecco

\begin{abstract}
ULTRASOUND-ACCELERATED THROMBOLYSIS: AN INNOVATIVE TECHNIQUE TO TREAT NATIVE ARTERIOVENOUS FISTULA ACUTE THROMBOSIS Abstract. Native arteriovenous fistula (AVF) acute thrombosis is the first cause of vascular access failure. Different procedures can restore AVF patency: pharmacological thrombolysis, mechanical thrombolysis and surgical thrombectomy. At the same time vascular stenosis must always be found and treated by angioplasty. Direct thrombolysis with endovascular catheter with or without mechanical thrombolysis is becoming the treatment of first choice. Ultrasound-accelerated thrombolysis by the EkoSonic Endovascular System (EKOS) is an innovative technique: a multilumen catheter releases low-dose ultrasound energy in addition to thrombolytic infusion inside the thrombus. EKOS thrombolysis is faster, more effective and less dangerous than standard thrombolysis. This technique brings neither thrombus fracture or breakage nor hemolysis, so it is better than mechanical thrombolysis. Ultrasound-accelerated thrombolysis is used to treat acute arterious and deep venous thrombosis and massive pulmonary thromboembolism. We first used microsonic accelerated thrombolysis to treat a $20 \mathrm{~cm}$ long thrombosis of a distal radio-cephalic fistula involving the forearm superficial venous circle. $E K O S \circledR$ system permitted a rapid resolution of vein thrombosis, restored the forearm superficial venous circle patency without any injury and allowed an immediate use of the AVF for dialysis. After 15 months, the AVF is still patent and well functioning.
\end{abstract}

Key words: Thrombolysis, Dialysis, Arteriovenous fistula, Ultrasound

Conflict of interest: None.

Financial support: None.

Ricevuto: 20 Novembre, 2012; Accettato: 30 Gennaio, 2013

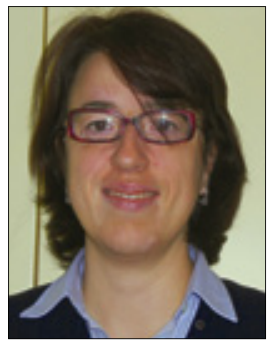

Laura Buzzi

\section{La trombosi della FAV con vasi} nativi

La fistola artero-venosa (FAV) con vasi nativi rappresenta il migliore accesso vascolare per emodialisi, perché riduce la morbilità e la mortalità dei pazienti (1). Per migliorare la sopravvivenza della FAV, occorre preservare l'integrità del patrimonio venoso e monitorarne periodicamente la portata, per individuare precocemente eventuali stenosi emodinamicamente significative, che sono la causa principale della trombosi (2-4). Le stenosi delle FAV native sono generalmente localizzate a livello dello "swing segment", ovvero nel tratto iuxta-anastomotico della vena mobilizzata per confezionare l'anastomosi artero-venosa. Le stenosi delle FAV radiocefaliche al polso si formano più frequen- temente nella zona iuxta-anastomotica, mentre per le FAV costruite al gomito le stenosi si localizzano soprattutto a livello della vena prossimale. È segnalato inoltre un numero crescente di stenosi sull'inflow, ovvero sull'arteria nel tratto iuxta-anastomotico: queste causano soprattutto un malfunzionamento precoce delle FAV, che presentano una maturazione lenta o insufficiente. Le stenosi sull'outflow determinano generalmente un malfunzionamento tardivo delle FAV, provocando una stasi venosa e una turbolenza di flusso che aumenta il rischio di trombosi. La correzione pre-emptive di una stenosi non è unanimemente raccomandata $(5,6)$. Alcuni studi suggeriscono che la variabile principale nel determinare la trombosi della FAV sia il timing dell'intervento: intervenire su una FAV trombizzata si associa a un rischio di perdere la FAV 8 volte maggiore rispetto al trattamento preventivo $(7,8)$. Le linee-guida KDOQI raccomandano di intervenire quando la portata è $<600 \mathrm{~mL} / \mathrm{min}$ o si riduce del $25 \%$ scendendo al di sotto di $1 \mathrm{~L} / \mathrm{min}$ per le $\mathrm{FAV}$ protesiche, 
mentre il timing di intervento per le FAV native è consigliato per una portata $<400-500 \mathrm{~mL} / \mathrm{min}(1)$.

\section{Come risolvere la trombosi}

In caso di trombosi acuta della FAV, occorre intervenire tempestivamente (preferibilmente entro 48 ore) con diverse procedure: trombolisi farmacologica, trombolisi meccanica o trombectomia chirurgica (1). La trombolisi meccanica, agendo traumaticamente sulla parete della vena, pregiudica l'integrità anatomica del vaso e, pur ripristinandone la pervietà, rischia di favorire fenomeni trombotici. La rimozione chirurgica del trombo, per motivi tecnici, rischia di alterare l'integrità del vaso e, generalmente, può riguardare tratti di vena non estesamente interessati dal trombo. La trombolisi farmacologica si avvale dell'infusione di farmaci fibrinolitici che agiscono sia localmente che in maniera sistemica e, pertanto, non è esente da complicanze emorragiche, anche maggiori. La trombolisi diretta mediante cateterismo endovascolare con o senza trombolisi meccanica sta diventando la terapia di prima scelta. Riassumiamo brevemente come si può svolgere il procedimento (esistono variazioni a seconda dell'esperienza del medico):

Esame fisico della FAV: ricercare segni d'infezione (controindicazione assoluta alla trombectomia).

Anamnesi: verificare se il paziente sia cardiopatico o pneumopatico: frammenti di trombo possono embolizzare nel circolo polmonare (pazienti con insufficienza cardiaca, ipertensione polmonare, shunt dx-sx sono da candidare a trombectomia chirurgica: il chirurgo clampa l'outflow venoso durante la procedura); altra controindicazione: revisione chirurgica entro 30 gg. Premedicare il paziente con ASA $325 \mathrm{mg}$.

Flebografia: valutare le vene periferiche e centrali; consigliata la puntura dell'arteria con un catetere multipurpose in direzione anterograda; in caso di estesa lesione venosa stenotica o multiple stenosi sequenziali, rischio di risultato non soddisfacente.

Rimozione del trombo: inserzione di introduttore 6-7F, bolo eparina 2,000-5,000 U; 3 tipi di tecniche: trombectomia mediante aspirazione o con pallone, trombolisi farmacologica, trombectomia meccanica; pervietà primaria a 3 mesi sovrapponibile per tutte le tecniche $(35 \%-60 \%)$; pervietà a lungo termine indipendente dalla tecnica e determinata dalla completa correzione delle stenosi (A e V) e dalla completa rimozione del trombo.

Trattamento delle stenosi significative: possibile dilatare prima la stenosi, per decomprimere la FAV e per testare l'elasticità della vena stenotica; in alternativa dilatazione dopo la trombectomia, perché la stenosi limita il trombo e riduce il rischio di embolizzazione periferica; palloni ad alta pressione $(>20$ atm) generalmente per stenosi venosi post-anastomotiche dei graft, con diametro $10 \%-20 \%$ più grande della stenosi; se stenosi tenace, cutting balloon.

Rimozione dell'occlusione prossimale all'anastomosi arteriosa: inserzione di un secondo introduttore nella vena in direzione retrograda; rimozione temporanea dell'introduttore arterioso, lasciando in sede il filo guida, per facilitare la rimozione del trombo periarterioso.

Rimozione del tappo di trombo con fibrina tenacemente ade- so alla parete arteriosa a livello dell'anastomosi, piccolo $(<5$ $\mathrm{mL}$ ), rischio minimo di embolia polmonare; generalmente si usa catetere da embolectomia over-the-wire Fogarty 4F.

\section{Controllo finale con fistolografia}

La rimozione del trombo nelle FAV con vasi nativi è una sfida tecnicamente più complessa rispetto alle protesi in PTFE, perché il trombo è più voluminoso ed esteso, la presenza di aneurismi favorisce la formazione di trombi cronici difficilmente rimovibili con tecniche percutanee e spesso esistono stenosi multiple, perianastomotiche o nei siti di incannulazione.

\section{Trombolisi Accelerata MicroSonica (TAM)}

Quando si forma un trombo all'interno di un vaso, i recettori del plasminogeno sono incastrati all'interno di una fitta rete di fibrina. Perché il trombo possa essere dissolto, gli agenti litici devono avere accesso a questi siti recettoriali. I filamenti di fibrina, saldamente intrecciati fra di loro, ostacolano la penetrazione del fibrinolitico, limitandone l'accesso ai siti recettoriali all'interno del trombo. La fitta trama di filamenti di fibrina può essere allargata dall'azione di ultrasuoni ad alta frequenza e bassa energia (2.2 MHz, $0.45 \mathrm{~W})$, emessi da un catetere posto all'interno di un catetere multilume di $5.4 \mathrm{~F}$ (Fig. 1). Il catetere esterno, denominato DDC (Drug Delivery Catheter), è lungo $106 \mathrm{~cm}$ e permette il rilascio del fibrinolitico all'interno del trombo. Il catetere centrale, denominato USC (UltraSound Core), contiene un dispositivo microsonico che emette radialmente impulsi intermittenti a varie ampiezze e periodi lungo tutto l'asse di trattamento. Burst intermittenti di ampiezza maggiore possono permettere all'energia ultrasonica di aprire in maniera più efficace la matrice del trombo, esponendo i siti recettoriali del plasminogeno e rendendo il trombo più permeabile al fibrinolitico. Il catetere USC può essere lungo 6, 12, $18,24,30,40$ e $50 \mathrm{~cm}$ : la lunghezza dell'USC viene scelta a seconda dell'estensione della trombosi da trattare. Attorno al lume centrale contenente l'USC è presente il lume di raffreddamento attraverso cui viene fatta scorrere soluzione fisiologica, allo scopo di ridurre il rilascio di calore dismesso dalle onde ultrasonore. Sensori di ottimizzazione della terapia consentono di modulare l'emissione delle onde microsoniche e di ottimizzare il trattamento. L'estremità prossimale del catetere è connessa all'unità di controllo, che genera le onde ultrasoniche e consente il monitoraggio continuo dei parametri di trattamento, in particolare della temperatura: l'unità di controllo riduce automaticamente la potenza degli ultrasuoni quando la temperatura si avvicina a $43^{\circ} \mathrm{C}$.

Attraverso il dispositivo endovascolare EKOS MicroSonic, il trombolitico permea in profondità all'interno del trombo sotto l'azione dell'energia ultrasonica. Studi in vitro confermano che l'assorbimento di t-PA all'interno del trombo esposto agli ultrasuoni supera del $48 \%$ dopo 1 ora e dell' $84 \%$ dopo 2 ore l'assorbimento di t-PA rispetto a un trombo non esposto ad ultrasuoni. Rispetto alla sola fibrinolisi, la TAM è più rapida (nelle trombosi venose e arteriose periferiche, il tempo di risoluzione si riduce del 40\%), più efficace (permea trombi di ogni forma, dimensione, volume ed età), più sicura perché riduce 
Fig. 1 - Dispositivi per la trombolisi accelerata microsonica.

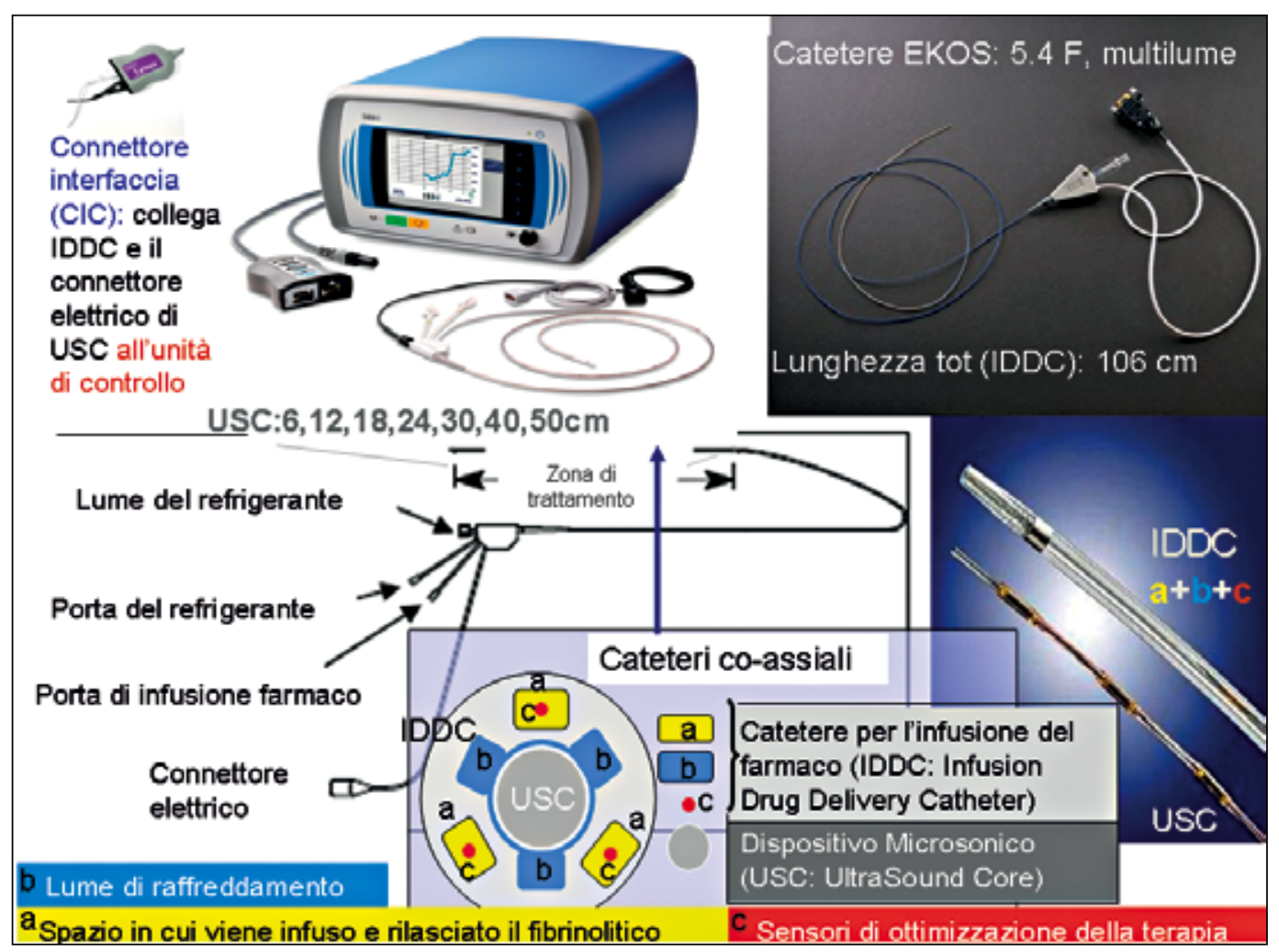

la dose di fibrinolitico. Rispetto alla trombolisi meccanica, la TAM ha un basso rischio di embolizzazione perché non agisce traumaticamente sul trombo e non provoca emolisi. Da alcuni anni la TAM è impiegata con successo nel trattamento delle trombosi arteriose acute, nelle trombosi venose profonde e nelle tromboembolie polmonari massive (9-12). Lin et al hanno osservato che nei pazienti affetti da tromboembolia polmonare massiva, la TAM risulta più efficace $(100 \%$ dei casi rispetto al $50 \%, \mathrm{p}<0.02)$, più rapida $(17 \pm 5.24$ ore vs $25.3 \pm 7.35$ ore) e più sicura (nessuna complicanza emorragica rispetto al $21.4 \%, \mathrm{p}=0.02$ ) della trombolisi semplice mediante catetere endovascolare (11). Parikh et al hanno osservato che nei pazienti affetti da trombosi venose profonde, il trattamento con TAM consente di dimezzare il tempo di trattamento e la dose di urokinasi infusa (dose totale di urokinasi 2 milioni vs 4.4 milioni di U; tempo di trattamento 19.3 ore vs 40.6 ore) (9).

\section{Trombolisi di una FAV mediante TAM}

Una donna di 61 anni, affetta da ipertensione arteriosa, iperparatiroidismo secondario e pregressa neoplasia mammaria in remissione da 7 anni, dializzata da 11 anni mediante una FAV radio-cefalica sinistra al polso, giunge al Servizio Dialisi con una trombosi dell'accesso vascolare occorsa da meno di 48 ore. All'esame con eco-color Doppler, si evidenzia un voluminoso trombo che parte subito dopo l'anastomosi artero-venosa latero-terminale della fistola e si estende all'interno della vena cefalica a monte dell'anastomosi, per un tratto lungo circa 20 $\mathrm{cm}$. Lungo la vena cefalica trombizzata sono presenti 2 grossi aneurismi, larghi $2 \times 2 \mathrm{~cm}$, distanti rispettivamente 7 e $18 \mathrm{~cm}$ dall'anastomosi, anch'essi occupati dal trombo (Fig. 2). Data la notevole estensione della trombosi, che coinvolge l'intero circolo venoso superficiale dell'avambraccio, abbiamo scelto di applicare, per la prima volta su una fistola per emodialisi, il trattamento con TAM.

\section{Inserimento endovascolare percutaneo del catetere}

La paziente è stata preparata per la procedura endovascolare percutanea eco-guidata. Sotto guida ecografica, è stato inserito un introduttore $6 \mathrm{~F}$ nella vena cefalica subito al di sopra dell'anastomosi. Nell'introduttore, è stato inserito un filoguida 0.025 " (può essere utilizzato anche un filo 0.035 "), che è stato fatto risalire oltre il lungo trombo all'interno della vena ascellare; sotto monitoraggio ecografico, abbiamo controllato che il passaggio del filo-guida non esercitasse traumi sul trombo potenzialmente emboligeni. Dopo un bolo di 5,000 U di eparina, il DDC è stato inserito all'interno del vaso lungo il filo-guida. Quindi abbiamo rimosso il filoguida e, al suo posto, abbiamo inserito all'interno del DDC il dispositivo centrale USC, con l'estremità distale oltre il trombo e quella prossimale completamente all'interno del vaso (il catetere ha 2 markers in platino, radio-opachi, all'inizio e alla fine della zona di trattamento); data la lunghezza dell'area da trattare, abbiamo scelto un catetere USC lungo $24 \mathrm{~cm}$ che abbiamo poi connesso all'unità di controllo. Infine, abbiamo connesso al DDC sia la pompa d'infusione dell'urokinasi diluita in soluzione fisiologica attraverso la drug infusion port, sia la pompa d'infusione di soluzione 


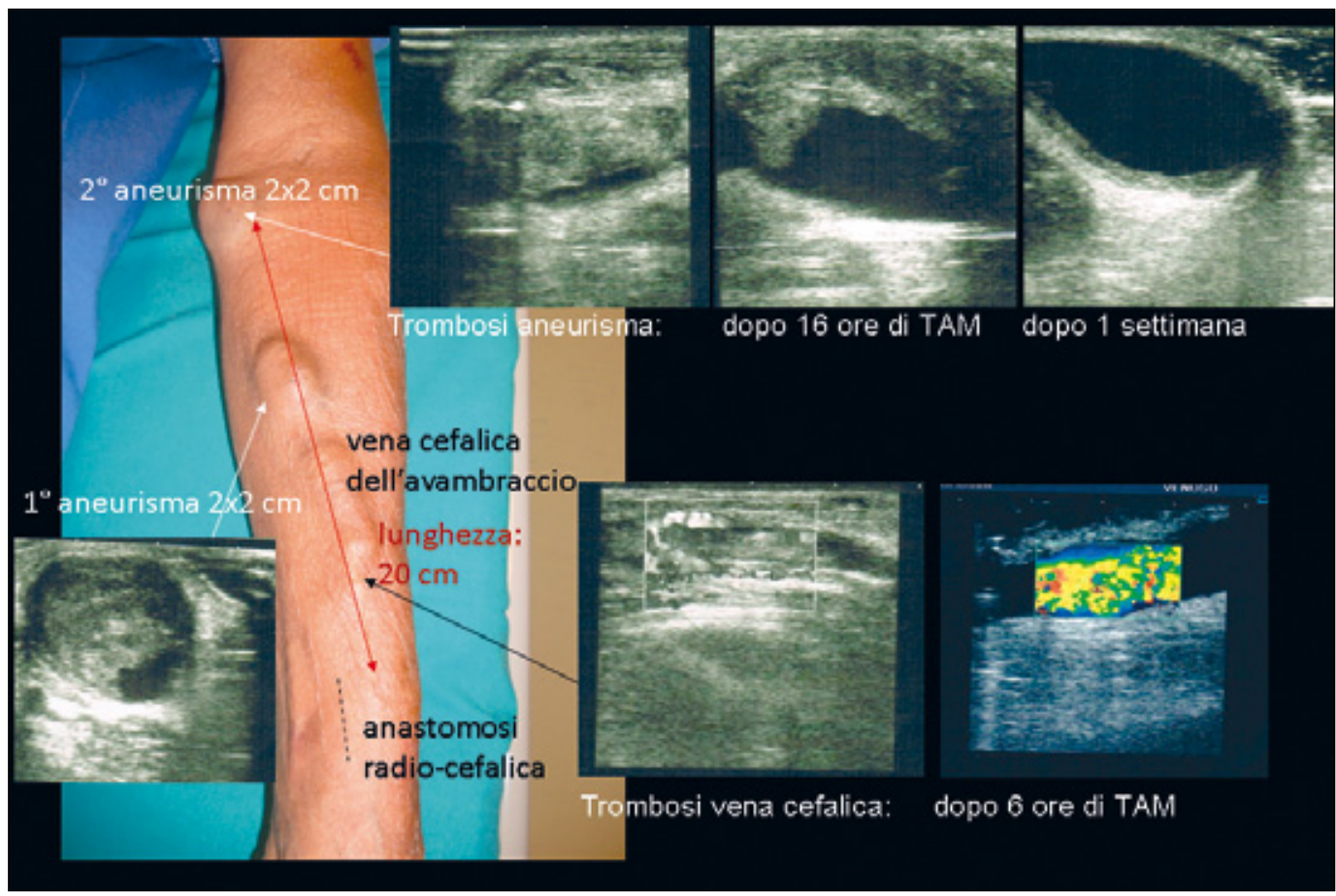

Fig. 2 - Trombosi della FAV, prima e dopo trattamento con TAM.

fisiologica alla velocità di $35 \mathrm{~mL} /$ ora attraverso la coolant port. Abbiamo iniziato la trombolisi associata all'emissione di ultrasuoni infondendo $75,000 \mathrm{U} /$ ora di urokinasi per 16 ore.

\section{Infusione dell'urokinasi in combinazione con l'emissione di energia ultrasonica all'interno della vena trombizzata}

Dopo la prima fase che necessita di un campo sterile, la paziente ha proseguito il trattamento nel proprio letto di degenza, senza nessun problema. Ogni 6 ore è stata sottoposta a controlli eco-Doppler (durante i controlli, è possibile interrompere momentaneamente l'emissione delle onde microsoniche che interferiscono con il segnale Doppler), per monitorare l'efficacia del trattamento ed eventuali complicanze.

Dopo le prime 6 ore, al controllo Doppler è stata osservata la completa ricanalizzazione del tratto di vena cefalica, lungo circa $9 \mathrm{~cm}$, compreso fra i 2 aneurismi. Dopo 16 ore, al termine del trattamento, il catetere e l'introduttore sono stati rimossi. Al controllo Doppler, è stata osservata una riduzione delle dimensioni del trombo all'interno dei 2 aneurismi: circa del 30\% nell'aneurisma distale e circa del $50 \%$ nell'aneurisma prossimale (Fig. 2). Tutta la procedura è stata ben tollerata e non si sono verificate complicanze. A 2 ore dal termine della procedura, la paziente è stata sottoposta a emodialisi utilizzando la FAV punta con 2 aghi; la seduta dialitica di 4 ore è avvenuta senza complicanze, con una velocità di pompa sangue di $300 \mathrm{~mL} / \mathrm{min}$, in assenza di ricircolo. La paziente ha iniziato una terapia anticoagulante ed è stata dimessa il giorno dopo la procedura.

Dopo una settimana, abbiamo osservato la completa rica- nalizzazione di tutto il tratto venoso in precedenza trombizzato: anche all'interno dei 2 aneurismi le apposizioni trombotiche erano completamente dissolte.

\section{Trattamento della causa della trombosi}

Dopo 2 settimane, il monitoraggio della FAV ha evidenziato una riduzione della portata al di sotto di $500 \mathrm{~mL} / \mathrm{min}$. L'esame angiografico della FAV ha evidenziato 2 stenosi emodinamicamente significative in prossimità dell'anastomosi $\mathrm{A}-\mathrm{V}$ : una a livello dell'arteria radiale, nel tratto peri-anastomotico; l'altra a livello della vena cefalica, nel tratto iuxta-anastomotico. La stenosi venosa è stata corretta mediante angioplastica con cutting-balloon con dilatazione fino a $4 \mathrm{~mm}$; la stenosi arteriosa è stata trattata mediante angioplastica con un pallone ad alta pressione $6 \times 40 \mathrm{~mm}$. Il risultato della procedura ha consentito di risolvere entrambe le stenosi e aumentare la portata della fistola attorno a $800 \mathrm{~mL} / \mathrm{min}$. Dopo 15 mesi, la FAV è pervia e funziona bene.

\section{Conclusione}

La TAM risulta efficace nel trattamento della trombosi vascolare acuta, perché consente una migliore penetrazione del farmaco trombolitico all'interno della formazione trombotica. Da alcuni anni è una terapia utilizzata nel trattamento delle trombosi venose profonde agli arti superiori e inferiori, dell'embolia polmonare massiva e delle trombosi arteriose periferiche; in letteratura non sembrano esserci segnalazioni relative al trattamento di trombosi degli accessi vascolari per emodialisi con questo tipo di procedura.

Quando avviene una trombosi acuta di una FAV, anche estesa, 
è possibile salvare l'accesso vascolare mettendo in atto tempestivamente tutti i provvedimenti diagnostici e terapeutici necessari per correggere la causa della trombosi e ripristinare la pervietà del vaso. Rispetto ad altre tecniche di rivascolarizzazione, la TAM offre alcuni vantaggi:

- è tecnicamente semplice, mini-invasiva e permette di effettuare in un'unica procedura sia l'angioplastica delle stenosi vascolari sia la TAM. Nel caso presentato la procedura di angioplastica è stata eseguita in un secondo tempo, in quanto l'individuazione delle stenosi peri-anastomotiche è avvenuta dopo la risoluzione della trombosi;

- rispetto alla sola fibrinolisi, la trombolisi è più rapida, con minori dosi di fibrinolitico e rischio emorragico ridotto;

- ripristina rapidamente il funzionamento della FAV, che può essere subito utilizzata per la dialisi;

- risulta efficace anche per trombosi di estese aree vascolari, ricanalizzabili e nuovamente accessibili per confezionare eventuali altri accessi vascolari;

- rispetto alla trombolisi meccanica, si accompagna a un basso rischio di embolizzazione periferica, perché non agisce traumaticamente sul trombo.

La TAM può essere una terapia efficace, utile e vantaggiosa anche nel trattamento della trombosi della FAV. Ulteriori applicazioni nel campo degli accessi vascolari emodialitici sarebbero utili per confermare ulteriormente la validità di questa tecnica.

\section{Riassunto}

Il trattamento della trombosi della FAV con vasi nativi, che è la causa più frequente della perdita dell'accesso vascolare, può avvalersi di diverse tecniche: trombolisi farmacologica, trombolisi meccanica e trombectomia chirurgica, cui associare la correzione della stenosi che ha provocato la trombosi. La trombolisi diretta mediante cateterismo endo- vascolare con o senza trombolisi meccanica sta diventando la terapia di prima scelta. La trombolisi accelerata microsonica (TAM) è un trattamento endovascolare innovativo: grazie all'emissione di ultrasuoni da parte di un catetere multilume che rilascia contemporaneamente il fibrinolitico all'interno del trombo, la TAM è più rapida, più efficace e più sicura rispetto alla sola fibrinolisi e, rispetto alla trombolisi meccanica, non è traumatica, ha un bassissimo rischio di embolizzazione e non provoca emolisi. La TAM, da poco impiegata nelle trombosi acute arteriose e venose profonde e nelle tromboembolie polmonari massive, è stata da noi utilizzata per la prima volta per trattare la trombosi acuta di una FAV radio-cefalica estesa all'intero circolo venoso superficiale dell'avambraccio per una lunghezza complessiva di $20 \mathrm{~cm}$. La TAM è risultata una tecnica semplice, ben tollerata e mini-invasiva che ha reso utilizzabile la FAV subito dopo la trombolisi. Dopo 15 mesi, la FAV è pervia e funziona bene.

Parole chiave: Trombolisi, FAV, Dialisi, Ultrasuoni

Dichiarazione di conflitto di interessi: Gli Autori dichiarano di non avere conflitto di interessi.

Contributi economici degli autori: Gli Autori dichiarano di non avere ricevuto sponsorizzazioni economiche per la preparazione dell'articolo.

\author{
Indirizzo degli Autori: \\ Dr.ssa Laura Buzzi \\ Nefrologia e Dialisi \\ Ospedale Bassini \\ Via Gorki 50 \\ 20092 Cinisello Balsamo (MI) \\ laura.buzzi@icp.mi.it
}

\section{Bibliografia}

1. NKF-DOQI Clinical Practice Guidelines for Vascular Access. Am J Kidney Dis 2006; 48: S176-S247.

2. Huber TS, Carter JW, Carter RL, Seeger JM. Patency of autogenous and polytetrafluoroethylene upper extremity arteriovenous hemodialysis accesses: a systematic review. J Vasc Surg 2003; 38(5): 1005-11.

3. Bittl JA. Catheter interventions for hemodialysis fistulas and grafts. JACC Cardiovasc Interv 2010; 3(1): 1-11.

4. Abreo K, Allon M, Asif A, et al. Which direction is right for vascular access surveillance? A debate. Nephrol News Issues 2010; 24(7): 30, 32, 34.

5. Paulson WD, Work J. Controversial vascular access surveillance mandate. Semin Dial 2010; 23(1): 92-4.

6. Paulson WD. Access monitoring does not really improve outcomes. Blood Purif 2005; 23(1): 50-6.

7. Allon M, Robbin ML. Hemodialysis vascular access monitoring: current concepts. Hemodial Int 2009; 13(2): 153-62.
8. Dossabhoy NR, Ram SJ, Nassar R, Work J, Eason JM, Paulson WD. Stenosis surveillance of hemodialysis grafts by duplex ultrasound reduces hospitalizations and cost of care. Semin Dial 2005; 18(6): 550-7.

9. Parikh S, Motarjeme A, McNamara T, et al. Ultrasound-accelerated thrombolysis for the treatment of deep vein thrombosis: initial clinical experience. J Vasc Interv Radiol 2008; 19(4): 521-8.

10. Owens CA. Ultrasound-enhanced thrombolysis: EKOS EndoWave infusion catheter system. Seminars in Interventional Radiology 2008; 25: 37-41.

11. Lin PH, Annambhotla S, Bechara CF, et al. Comparison of percutaneous ultrasound-accelerated thrombolysis versus catheterdirected thrombolysis in patients with acute massive pulmonary embolism. Vascular 2009; 17(Supp 3): 137-47.

12. Wissgott C, Richter A, Kamusella P, Steinkamp HJ. Treatment of critical limb ischemia using ultrasound-enhanced thrombolysis (PARES Trial): final results. J Endovasc Ther 2007; 14(4): 438-43. 